\title{
A New System Engineering Methodology Coupling Formal Specification and Performance Evaluation
}

\author{
J. Martins J.-P. Hubaux \\ Swiss Federal Institute of Technology \\ TCOM Laboratory, Telecommunications Services Group \\ EPFL-DE-TCOM-GST, CH-1015 Lausanne \\ martins@tcom.epfl.ch and hubaux@tcom.epfl.ch
}

\begin{abstract}
This paper proposes a new methodology for system engineering. It provides an integrated approach covering formal specification and performance evaluation. Therefore it associates the advantages of formal techniques that allow formal proof and automatic implementation with the benefits of performance evaluation. The basic idea is to abstract the relevant features of the system in the formal specification (structure, functional description), enhance it with relevant information for performance modeling (quality of service, operating load, processing architecture and processing constraints), and map them into a performance evaluation environment. In this paper, we develop the suitable extensions for the formal language SDL and apply them to an example: Transmission Control Protocol (TCP).
\end{abstract}

Keywords: System Engineering, Formal Specification, System Analysis, System Design, Performance Evaluation, Simulation.

\section{Introduction}

Performance is becoming a critical aspect of emerging broadband technology. Therefore, the use of powerful performance modeling techniques becomes of prime importance. Their goal is to obtain some notion of how the system will perform under a given set of conditions. Besides, they facilitate analysis of proposals for new services or technology because they are more tractable and less expensive than field trials for evaluating alternatives. Performance evaluation facilitates comparing alternative designs and finding which is the best according to the chosen set of criteria. Even if there are no alternatives, it helps in determining how well the system would perform and whether any improvements need to be made.

Formal description techniques intend to describe the structure and the behavior of systems. Their purpose is to produce correct and unambiguous descriptions, that should ultimately produce efficient implementations. The input to the formal specification is a set of requirement statements and a conceptual overview of the proposed system. The output is a formal representation that captures the functional description of the proposed system. A formal specification should abstract from implementation details in order to give an overview of the system, to postpone implementation decisions, and to allow all valid implementations. It makes use of neither design nor implementation concepts; it defines rather a model that represents the significant properties and functionality of the system, which can be controlled through validation, verification and conformance testing.

Traffic and queueing theorists have long been using protocol specifications to make a heuristic model of the system and then analyzing the model. When system 
specifications exist, the question arises whether a more direct path to performance analysis cannot be found. Our scope is to provide a methodology that steadily integrates formal specification and performance evaluation (Figure 1). Formal specification handles the logical consistency of the system than performance modeling handles the operational consistency of the system. An efficient system should be logically error free, but it should also be performing enough to achieve its service.

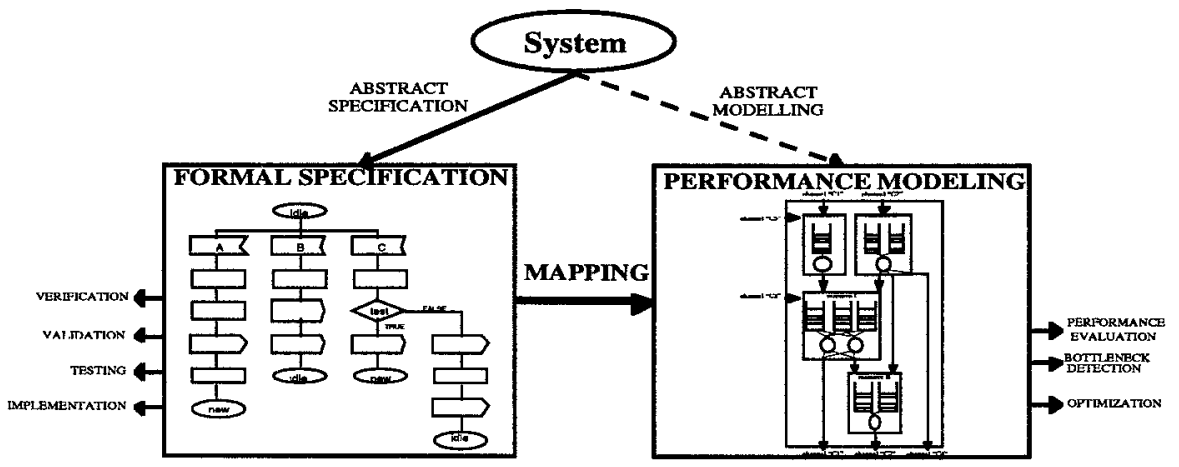

Figure 1: Performance evaluation and formal specification integration

Our methodology appeal consists in taking benefice of the similarities between the formal specification and the performance modeling. Both techniques share some common modeling that can be reused with each other.

Moreover, combining the formal specification and the performance evaluation allows to verify and improve the efficiency of the system. Formal specifications provide the basic input for the system validation, verification, automatic implementation and conformance testing. Therefore reusing a formal specification saves time because it reuses the expertise developed for formal modeling, and it avoids modeling errors because formal specifications can certify the logical consistence of the system.

\section{System Engineering}

System engineering aims at handling the complexity of systems in an organized way. Achieving this purpose requires supplying a methodology working at different levels, each focusing on certain aspects of the system.

- The analysis consists in understanding and modeling the system and the domain within which it operates; it includes abstracting important real-world features, focusing on what has to be done, independently of how it is done.

- The design consists in providing a high-level strategy for solving the problem and building a solution. It includes decisions about the organization of the system into hardware and software components and major policy decisions.

The implementation discusses the specific details for building the system.

Classical system engineering methodologies support analysis-design-implementation approach; however in many cases, analysis is refined in two models (Figure 2).

- The requirement specification aims to delimit the system, define the functionality of the system, and capture the customer requirements. It includes the definition of the service interfaces (which services, what quality of service, which cost) and of the information exchanged for a given service (which messages, which workload). This model is easy to understand and formulate from both the customer and the provider 
perspective, so they can talk to each other and see if we are building the correct system. This control is usually referred as the verification process.

- The analysis specification aims to structure the system independently of its implementation environment. It consists of the specification of the information held in the system, of the behavior that the system will adopt and of the details from presenting the system to the outside world. It is formed from the requirement specification. During the analysis, the system will be entirely partitioned into objects to obtain a robust and extensible structure. In practice it means that the functionality should be allocated to different components. When the system contains a large number of components, it is seldom possible to get a clear overview, so they are structured in subsystems. The task of a subsystem is to package the components so that complexity is reduced. The subsystem also works as handling units for the organization; all components that have a strong mutual functional and geographical coupling will be placed in the same subsystem. The analysis specification serves at least three purposes; at an early stage to specify, validate and verify the functionality and the system properties, then to provide a firm basis for design and implementation, and after implementation, to document the functional properties.

- The design specification aims at adapting the analysis specification to a particular implementation environment. The analysis specification was developed assuming ideal conditions that we must at this stage adapt to reality. The abstract vision of the analysis specification is suited because it provides a generic structuring that serves as basis for many alternative designs, and because it avoids the complexity introduced when looking at the implementation. On the other hand, design specifications provide physical designs of the system that will implement the specified functionality. This can be seen as mapping the abstract system defined in the analysis specification to a concrete system made up of hardware and software components. The design specification is an abstraction of how the system really is built. Its final structure will reflect how the implementation environment has affected construction.

- The product implementation consists of the software and hardware realization. It is the tail end of the system development; it provides the final product that will be used by customers. It should therefore satisfy and conform earlier phases of the system development. The control of its conformance towards the original analysis specification is usually referred as the testing or conformance testing process.

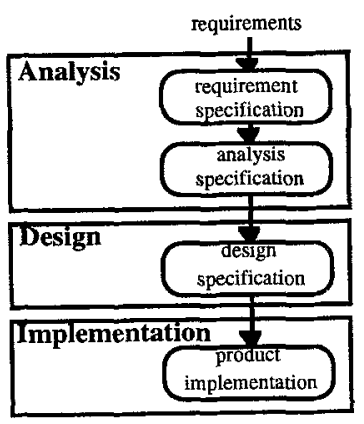

Figure 2: Classical system engineering approach

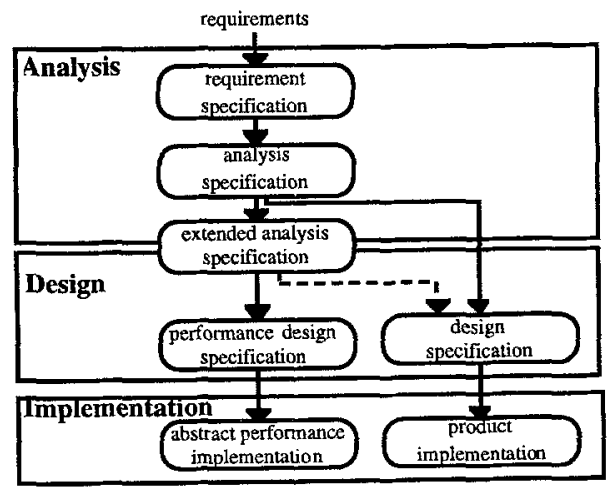

Figure 3: Our methodology decomposition 


\subsection{Our System Engineering Approach}

Our approach is organized according to the basic decomposition in three planes: analysis, design and implementation, for which we define seven models (Figure 3).

- The extended analysis specification aims at refining and integrating some of the topics usually covered by the design specification. The additional features are given in a highly abstract notation; that is, we are not interested to really apprehend the nature of the implementation but only those features that affect the non-functional aspects of the system (e.g., performance, cost, user perception). In classical system engineering, improving performance requires simulating the critical parts of the system, or performing prototyping to get an early opinion of what the system will look like, but this is a risky method since we can easily do unrealistic simplifications. Prototypes and simulations always aim to highlight particular issues. General conclusions on other issues therefore cannot be drawn from a prototype, nor from a rudimentary simulation that do not highlight these issues. Using an extended analysis specification has the advantage to combine the robustness of the analysis specification and to be close enough from the design to cover almost all the issues of the system. Moreover, computing the performance evaluation at that abstract level allows comparing alternatives of designs.

- The performance design specification aims at adapting the extended analysis specification to a particular performance evaluation environment. This can be seen as mapping the abstract model defined in the extended analysis specification to a concrete system made up of software.

- The performance abstract implementation provides an executable software that mimics the behavior of the system. Executing it would provide statistical results that once being analyzed bring to an end optimization of the system. Simulations promote identifying bottlenecks and determining critical performance parts of the system that require further design or specification.

\subsection{Our System Engineering Methodology}

Our methodology integrates methods from the formal world and from the performance evaluation world. Formal methods are used for the requirement specification, the analysis specification, the validation process and the verification process, then the performance evaluation methods are used to determine some of the non-functional properties (speed, accuracy and availability) of the system. We also provide a support for the interaction between those methods (Figure 4).

Our methodology starts from the customer and operator requirements. The customers what they expect from the system in terms of service, while the operators define how they intend to organize the system and what type of service primitives the system will offer. The requirement specification plays the role of a contract between the operator and the customers that clearly states the role of each part and their expectation in terms of services and interfaces. The scope of the requirement specification is not to define detail, but to define the essential properties or attributes required by the system environment. We shall make a distinction between functional and non-functional requirements. Functional requirements are concerned with the services and are inputs for the analysis specification, while non-functional requirements deal with implementation constraints and are inputs for the design specification.

Usual methods to conceive earlier iterations of the requirement specifications are natural languages, i.e., English, French, ... However, to capture and to analyze needs 
and expectations, so that they can be mapped in rigid requirement formulas, involves using formal methods. Nevertheless, before formalizing a requirement specification, it can be iterated as many times because expressing the requirements helps to understand the needs better. The formalization of the requirement specification enjoins using a formal method. There are many formal methods available nowadays, however, the most common ones are those standardized: SDL (ITU), LOTOS and Estelle (ISO).

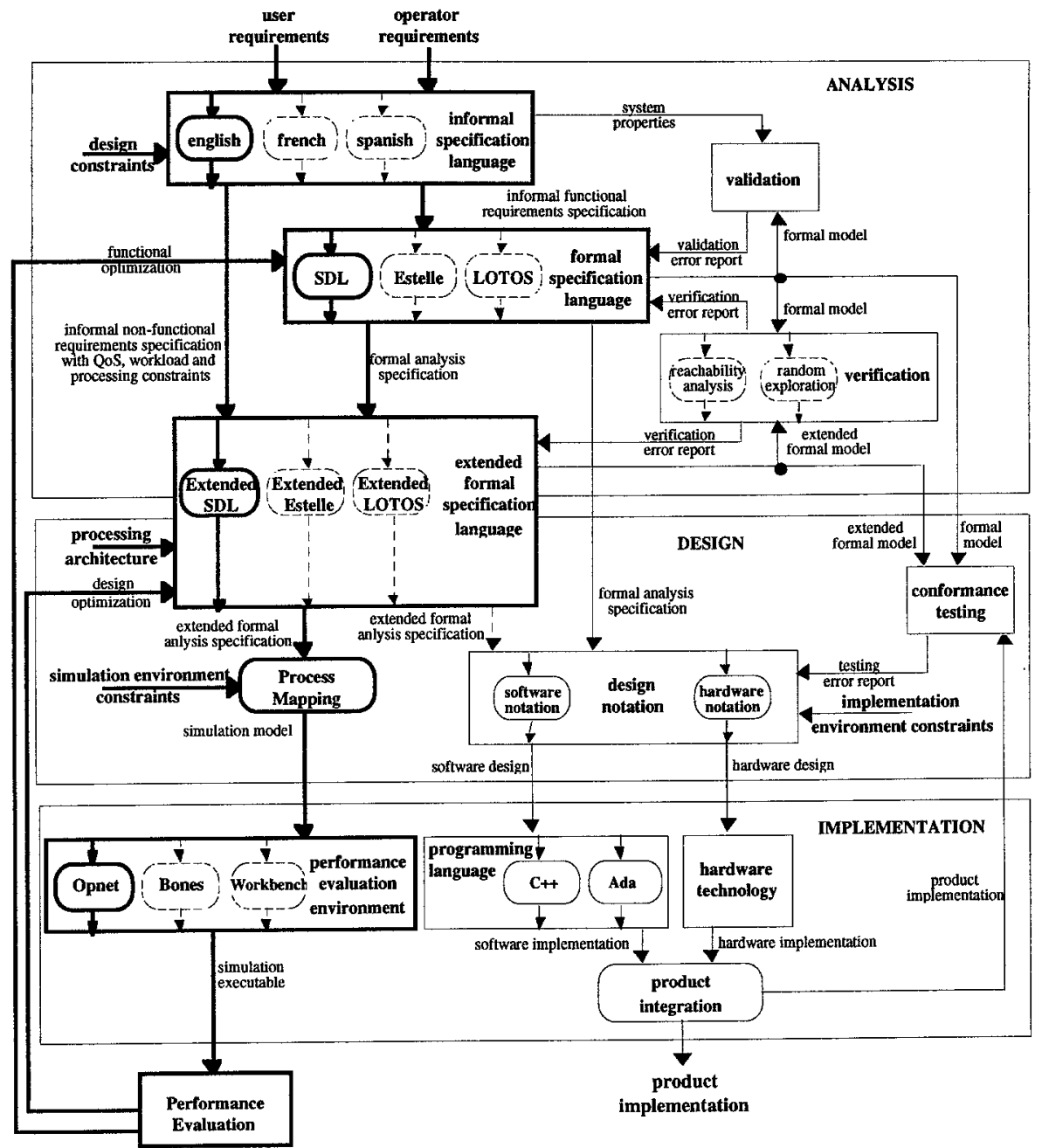

Figure 4: Integration methodology

Formalization provides a model which by successive refinements will meet the analysis specification, and which significant properties and functionality can be verified through validation and verification. As there is no clear agreement on these notions, we define them according to [20]:

* The verification is the process that compares a formal specification with the specification of what the system is intended to do, namely the services and system properties that the system provides to its users. A proof is performed to verify that 
behavior of the system corresponds to its required properties; however, the verification is an informal control that checks the general structure and property assertions of the requirement specification, and puts them in confrontation with the informal requirements of the customers.

* The validation is the process that increases the confidence in the correctness of the system. The validation process checks if a formal specification is logically consistent to a set of correctness criteria. The behavior of a verified model is defined by the set of execution sequences it can perform (formally defined as an ordered finite set of states, these latter formally defined as the specification of all local and global variables, all flow control structures of processes, and the contents of all communication links). In the literature, the correctness properties are often classified in two broad classes: safety and liveness. Intuitively a safety property asserts that "nothing bad happens", and a liveness property asserts that "something good will happen". Liveness properties are in some sense dual to safety properties; to state that something is inevitable, we can state that all deviant behaviors are impossible. Usual safety properties in a telecommunication environment assert the absence of deadlocks (a state in which no further execution is possible), of unspecified receptions (a signal arrives when the receiver does not expect it or cannot respond), of livelocks (execution sequence repeated indefinitely often without making effective progress), and of assertion violations (correctness criterion expressed by a boolean condition that must be satisfied whenever a process reaches a given state).

Since a formal model provides a safe representation of the system, it is a good basis for starting design and implementation. One possibility is to continue working on the formal analysis specification, even when incorporating the design. However, this is not suitable. When further developing the product, the analysis specification is needed to reason about when to incorporate the changes, since it has far less complexity than the design specification. Evenly, it is more interesting to develop a new specification for the performance evaluation that integrates and abstracts the non-functional behavior, instead of using the design specification because one important issue when simulating systems is avoiding inappropriate level of details. A more detailed simulation requires more time: the implementation is longer to develop, the likelihood of bugs increases and it becomes harder to spot them, and it also requires a grater computer time to run; this without necessarily improving the level of confidence of the simulation results because a detailed model may require more detail knowledge of input parameters, which, if not available, may make the model inaccurate. Moreover, at reusing the design specification, we loss the benefit of the formal validation. Thus, we have decided to split the tail of the system development methodology in two branches, one oriented to performance evaluation and one oriented to product implementation. The implementation branch allows modifying the semantics of the analysis specification. There are no rules how these modifications are performed and there are no assertions about their influence and limitations on the functional behavior. On the other hand, the performance evaluation branch remains consistent with the original formal analysis specification semantics.

Each design alternative may be characterized by non-functional properties such as execution speed or memory requirements. Such properties may be important for the system performance. Non-functional properties should therefore be put dawn in the extended analysis specification, to ascertain that the right kind of design is chosen from the functionally equivalent alternatives. In order to conserve the benefit from the formal analysis specification we should describe these design alternatives within a formal method. Unfortunately nowadays current formal methods lack of expressive 
power to describe non-functional properties. However, equivalent developments can be done for the other languages. Therefore, it is necessary to extend the formal method with a quantification of the interaction between the system and its environment (workload), a definition of the performance settings (quality of service), a description of processing resources, and a description of the processing constraints. The extended formal method should conserve the original semantics, and when no other solution is available modify it, but limiting its effect on the original method. Our approach is generic, nevertheless, in further developments, we will focus on the extension of SDL and we will provide the methodology according to the particularities of this language.

The extended analysis specification abstracts from real implementation considerations, but it includes information that clearly belongs to design. For this reason, we have located the extended formal methods in an intermediate position, half in the analysis and half in the design. Its input is the analysis specification and its output is an extension that embraces part of the design. Its output has been steered for performance evaluation purpose, but it can also be used as input for the design specification.

The performance design specification adapts the extended analysis specification to a performance evaluation environment. There are many tools available nowadays, however, we choose OPNET because its structure is close to the one of SDL.

The performance abstract implementation provides an executable software code that has to be computed in order to provide some statistical results that should further be analyzed. The performance analysis is a complex task that requires, first to develop a set of performance experiments that allow to evaluate the selected metrics, then to acquire high confidence on statistical results by running simulations, and finally to develop a systematic analysis method that allows to determine bottlenecks and to provide them as outputs for functional and design optimizations, in case the system fails to satisfy the performance requirements.

On the other branch of the methodology, the design specification translates the analysis specification in a set of suited formalisms for the implementation taking in consideration the particularities of the system under design. The achieved set of models is then implemented as a set of independent components that are aggregated to achieve the final product. The product should still be checked for conformance. Testing consists in comparing the implementation with its analysis specification and determining if the product respects the original statements made about the system.

\subsection{Related Work}

Analyzing the quantitative performance and establishing the functional correctness of communication protocols directly from their formal specification has long been an objective of research in protocol engineering. Most efforts to date have concentrated on verifying [7], validating [20], and testing [16] functional correctness. Nevertheless, performance evaluation is equally desirable to derive measures such as potential throughput, response time, or to determine bottlenecks. The performance of a communication system is a complex interaction between the physical processing resources employed, the protocols involved and the workload on the system. A performance model should take all these factors into consideration. Furthermore, quantitative analyses involve time, and formal specification standards (Estelle, SDL, LOTOS) originally left the concept of time open to interpretation. Much effort has been invested during the last decade in the area of integrating time, probabilistic information and processing description in formal specifications. Many performance 
models are based on existing algebraic model extensions: some concern time $[14,12$, $2]$ and some concern stochastic extensions $[15,10,18]$.

There are also many extensions applying on finite state machines. For instance, in [3], authors propose an extension of the language Estelle that allows the description of performance aspects as time delays, resource usage and stochastic behavior. They introduce the concept of resource and associate it to process instances. They also define a delay clause that forces the process to hold for a duration before to complete a transition and provide them with a stochastic attribute. Finally they introduce the principle of process interaction through queues and associate a service delay to each queue. In [22], authors propose a hybrid model combining EFSM and QN. They enhance the ESTELLE specification by specifying transition time and by adding a set of external variables that describe the resource demands. In [11], authors present a method to derive semi-Markov models of the protocol execution directly from its state transition graph. In [5], Dembinski suggests how the Estelle semantics may be reinterpreted within a queueing network. In [1], authors propose an extension of SDL, called Timed SDL or TSDL. The principle is to extend SDL transitions with stochastic and time information. In [9], the proposed method enhances the formal specification with performance submodels which finally yield to a combined model that is quantitatively assessable and encloses the original formal description. The principle of this method is to enhance EFSM with "places" that serve as interfaces to the environment. They assume input places for message to be received and output places for messages to be forwarded. Places may be limited in capacity. The idea behind the introduction of places is to connect each input place to the output of a queueing network and to connect each output place to the input of a queueing network. Performance evaluation is then performed on the basis of the queueing network obtained by this association. In [17], authors consider an extension of SDL incorporating the concepts of mission, resource, delay, scheduling mechanism, and random decision. In [21], Wohlin proposes a methodology that transforms SDL descriptions into descriptions combining formal description and performance evaluation. In [8], the authors propose a method to extend a formal specification, based on finite state machine, with the description of the traffic source and a queue that serves as the interface between the source and the protocol. This queue is designed with a capability of storing messages that the protocol cannot immediately transmit, thereby smoothing the message flow but potentially introducing delay.

Other specification formalisms have also been proposed in the literature. In [13], author proposes a method to capture the Quality of Service or Network Performance requirements. The principle of the method is to map SDL specifications to a global state transition system. Then states and transitions are described in terms of logic formulae over state positions. In [6] authors present the most important classes of nets, gives their analysis possibilities and shows how they can be used to model and analyze communication and cooperation protocols.

\section{Applying the Methodology to SDL}

\subsection{SDL - Analysis Specification}

The basic idea of SDL [19] is to describe a system as communicating processes. A process is an extended finite state machine (EFSM), that is either processing data or if no data is available for processing, is dormant in a state (the state defines what actions a process is allowed to take, which events it expects to happen, and how it will 
respond to those events). When data becomes available the machine starts processing and continues until all the possible processing has been accomplished, then it goes dormant in the same state or in an other one. The processing performed depends solely on the state in which the machine was last dormant, on the data that becomes available and on the local conditions. Several items of data may become available during the machine processing of previously available data, and a queue is associated with the machine to pile up the data as it arrives. The usual representation is by means of a graph consisting of nodes, each indicating a state and arcs, these later indicating transitions. Each transition has a trigger condition to indicate the data and local conditions that if available, would activate the transition.

Communication between processes is performed asynchronously, by way of connection paths (channels or signal-routes), by discrete signals. A system contains blocks interconnected by way of channels. The channels are the links through which the blocks communicate with each other or with the environment. A channel can be unidirectional or bi-directional. A bi-directional channel can be considered as two independent unidirectional channels. The channels are typed, meaning that they can only contain messages of certain types called signals. A block can contain either a substructure of blocks, or one or several processes interconnected by signal-routes. The signal-routes are the links through which processes can communicate with each other inside a block, and with the block boundary.

In SDL a concept of time is defined. All processes in the system may acquire the value of the current real time. Furthermore, to model time-outs, SDL is equipped with timers; the timer mechanism stimulates a process as a function of the defined time by placing a timer signal in the input queue of the process.

\subsection{Extended SDL - Extended Analysis Specification}

Performance Evaluation requires a consistent representation of the system under analysis to achieve a confident evaluation. For this reason, the first question that we should answer when reusing an SDL specification for performance evaluation purpose, is to determine the level of confidence of the model. SDL is composed from abstract communication media and extended finite state machines, while a real-system is composed from physical components and communication networks. Consequently, the SDL representation sustains some differences towards the real-systems; some differences concern the nature of the components, than some address the functioning of the components. Therefore creating a consistent representation that should be reused for performance evaluation should eliminate as much of these differences as possible. It implies defining some SDL extensions that restrict the scope of these differences.

\subsubsection{Modeling Processing Time}

A basic assumption in SDL is that the system is fast enough to process the offered load. In a real-system this assumption is disabled because each signal transfer and each transition of a process take some time and require some processing resource. To map the formal SDL model, the real-system should be fast enough to meet the traffic load and response time without destroying the validity of the SDL descriptions.

Extended SDL provides a new transition concept that specifies processing duration. Each transition specifies zero, one or several delay clauses that force the transition to pause and model processing duration. The associated execution model stipulates that all the actions before a delay clause are executed immediately, then the execution is 
suspended for the specified duration; when completed, the automaton resumes its execution until the next delay specification.

$<$ delay $>::=$ delay $<$ delay identifier $\left(\{<\text { delay item }>\}^{*}\right)<$ end $>$

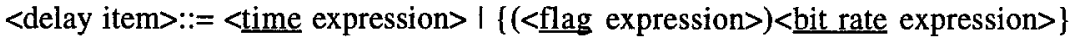

A delay specifies the pause duration during a transition. A delay may be expressed in seconds (<time expression $>$ ) or as a bit rate (<bit rate expression $>$ ), in which case the duration is calculated as the ratio between the signal length and the bit rate. In this latter case a flag should be set to TRUE. Each delay is identified by a name that distinguish it from other delay statements in the same process.

Another important performance evaluation feature that is not considered within SDL specifications is the specification of the physical distance covered by a communication link. In reality however, there may be large physical distances. This means that, in real systems, the transmission of information between two equipments may be delayed. Therefore, Extended SDL specification provides a special type of channels that allow modeling propagation delays.

All the temporal extensions of Extended SDL remain consistent with the SDL formal model. During the validation of the SDL model we will check the sequence of states and events of the system. Each validation state is defined by the aggregation of the state values of all the processes, of the content of all the communication links and of the variable values. However, there is no variable indicating the current time, that is, the functionality is verified independently of the time. In that sense, an Extended SDL specification is identical to the SDL specification because the sequence of events and states remains unchanged.

\subsubsection{Modeling Unreliability}

SDL systems may suffer from specification errors, but the abstract representations they provide do not suffer from physical errors. SDL assumes that processes and communication links always operate according to their specifications. It is not assumed that processes will stop or that communication links will distort the content of signals. But in the real world, errors manifest themselves as faults in operation of processes and communication. Hardware errors, physical damage and noise are caused by physical phenomena outside the realm of SDL. However, their effect is often handled explicitly in SDL specifications. One must consider what may happen, how it can be detected and how damages may be limited. One must consider what a process should do if it never gets a response to a request or if it gets an erroneous response.

Since communications have a considerable influence on the performance, it is vital to provide a realistic model of the communication links. In SDL, communication links (channels and signal-routes) convey the sequence of signals from the initiating port in that order to the end port, without introducing any distortion to the conveyed information. In channels, the transfer delay is non-deterministic (default) or negligible (no-delay); signal-routes always have a negligible delay. From a performance evaluation point of view, negligible and non-deterministic delays are unsuitable because performance evaluation requires an exact knowledge of the temporal operating. Besides, in real world, communication links often suffer from disfunctionments and errors that make information to contain errors or to be lost. Fortunately, SDL allows refining communication channels and modeling unreliability by means of channel substructures. However, proper handling of this aspect can be very complex and normally requires additional functionality in the SDL specification. There is no 
analogous mechanism to model unreliable signal-routes. Nevertheless, in SDL only the interfaces between the system specified and its environment are mandatory; all internal structures are purely a means to express the overall system behavior. Thus, specifying unreliable signal-routes is achieved by modifying the internal structure of the system and transforming the signal-route into a channel.

During performance evaluation, communications links are frequently specified as unreliable. For this reason, we decided to specify a macro that facilitates their specification. An unreliable channel macro may be used instead of a channel definition. When expanded, it should be seen as a channel substructure. Each direction is specified by a single process that generates errors and losses according to the specified distributions and that delays the signals by <time expression $>$ units of time. $<$ unreliable channel macro>::= channel <channel name> [nodelay] <unreliable path> [<unreliable path $>$ ] endchannel $[<$ channel name $>]<$ end $>$

<unreliable path>::= from <channel endpoint> to <channel endpoint> with <signal list> $<$ end $>$ [transferdelay <time expression $><$ end $>$ ] [error <distribution definition $><$ end $>][$ loss $<$ distribution definition $><$ end $>]$

\subsubsection{Modeling Processing Resources}

The model of concurrence used in SDL assumes that processes behave independently and asynchronously. There is no relative ordering implied by the sending and reception of signals. This permits SDL processes to be implemented either truly in parallel on separate hardware or in quasi-parallel on shared hardware. Moreover we may distinguish between two ways of executing parallelism: the synchronous operating, where operations are performed under the control of a common clock, and asynchronous operating, where operations are performed independently and possibly at different times. In the other hand, quasi-parallelism implies that only one process is active at a time and that the active process will block operating of other processes as long as it is allowed to remain active. This will affect the response times of the blocked processes, and it remains an acceptable approximation of the SDL semantics as long as the delays it introduces are acceptable to the environment. This will affect the response times of the blocked processes, and it remains an acceptable approximation of the SDL semantics as long as the delays it introduces are acceptable to the environment. Quasi-parallelism implies a scheduling algorithm that is handled by an operating system. The SDL model should represent it as a layer that implements a quasi-parallel virtual machine on top of the physical machine.

The act of aligning the operations of different concurrent processes in relation to each other is generally called synchronization. Such alignment is necessary not only to achieve correctness in communication, but also to control the access to shared resources. In SDL synchronization is achieved by means of buffered communication in which the sender may produce infinitely many signals without waiting for the receiver to consume them, which mechanism is often referred as asynchronous communication. Therefore SDL synchronization is time independent, using an asynchronous medium with infinite buffer capacity. In practice however, the queue will be finite, so in the case of a full queue the sender will have to wait or signals will be lost. To avoid it, output from an SDL process must be delayed until the receiving buffer is ready. This deviation from the SDL semantics can hardly be avoided in a finite implementation; care is needed to reduce to a minimum the practical problems this may cause. The synchronization of SDL rests on a basic synchronization mechanism called mutual exclusion. Only one process at a time can 
get access to the input queue. Only one service at a time can get access to shared data within the process and only one process at a time gets access to reveled data. Mutual exclusion is generally needed in the access to shared resources.

SDL has an unbounded queue in the input port of each process and allows infinite data to be specified. Hence the designer must find ways to implement potentially infinite SDL systems using finite resources. In practice however, all resources in a real system are finite. They may be a maximum number of processes the operating system can handle or a maximum number of buffers for sending messages. The memory space is finite and even primitive data like integers are finite. This deviation from the SDL semantics can hardly be avoided in a finite implementation; care is needed to reduce to a minimum the practical problems this may cause.

SDL processes consist of a single processing resource with a single infinite queue that follows a FIFO discipline. Even though this modeling is sufficient to capture the nature of many processes, it does not hold for all real processes. Thus, Extended SDL provides a new model of processing architecture. The idea is to associate each process with a set of finite queues and a set of servers (Figure 5).

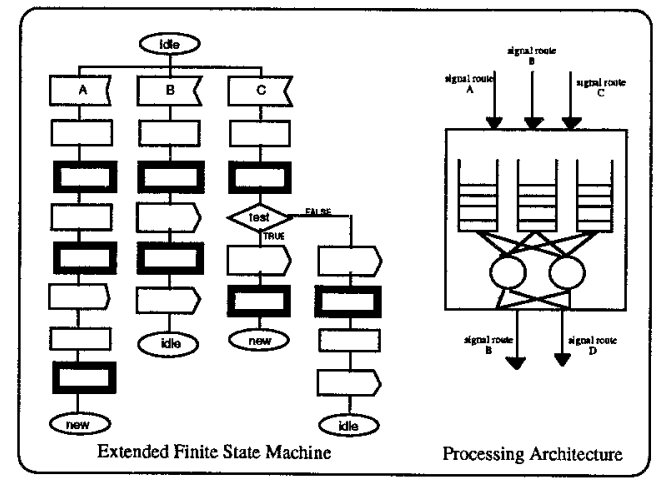

Figure 5: An Extended SDL process specification.

$<$ process definition $>::=$ process $\{<$ process name $>$ < process identifier $>$ \} $[<$ number of process instances $><$ end $>$ ] [ $<$ formal parameters $><$ end $>$ ] [<valid input signal set $>$ ] $[<$ resource definition $>]$ [<process body $>$ ] $\{<$ entity in process $><<$ signal route to route connection $>\}^{*}$ endprocess $[<$ process name $>\mid<$ process identifier $>]<$ end $>$

From a functional point of view, our extension may be seen as a macro that when expanded replaces a process by a set of processes (one for the modeling of the access, and a set for the modeling of multiple servers) (Figure 6). The access process manages the access to service of external signals, while each functional process models the functional behavior of the process specified in SDL. Thus, the extension may be seen as a modification of the specification but not as a modification of the semantics.

The access process defines where and how arriving messages should be stored. For instance, a process handling three priority data streams, can store them in three queues. Each signal arriving to the process will be analyzed and depending on its type and its parameters (associated variables) it decides in which queue it will be stored.

If an Extended SDL process specifies several servers, the expanded model instantiates the same number of process instances, each driving its server. At the completion of a transition, each process indicates to the access process that it is ready to receive the next signal by means of an exported variable. 


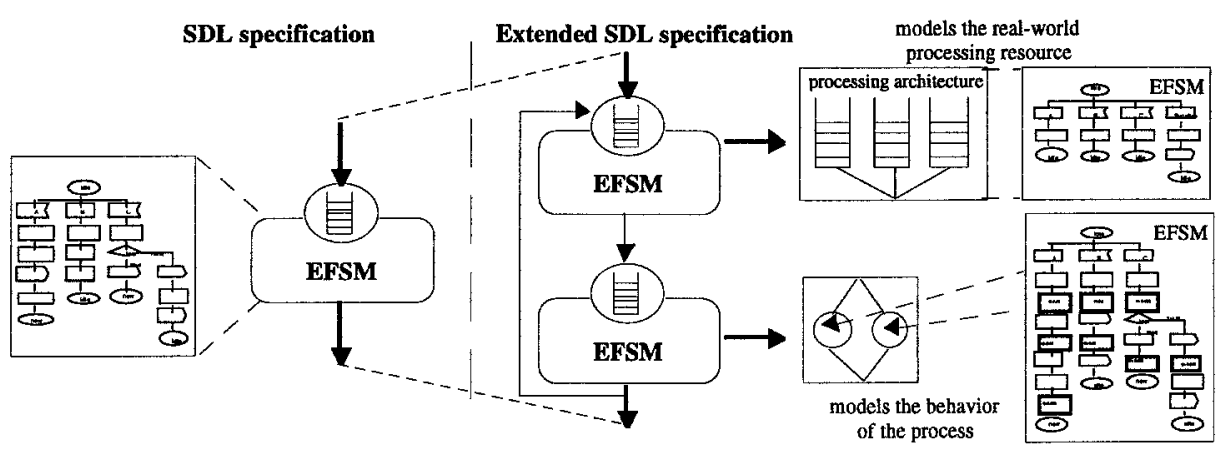

Figure 6: Extended SDL processing model

Our extension is based on existent SDL constructs. Therefore, we guarantee that we do not modify the semantics of SDL.

$<$ resource definition $>:=$ resource $<$ process name $><$ end $>$ \{ $<$ queue definition $><$ server definition $>\}+$ endresource $[<$ process name $>]<$ end $>$

Each processing resource can be seen as customers arriving for service, waiting for service if it is not immediate and leaving the system after being served. It is divided in four components (Figure 7): input, queueing, server, and output.

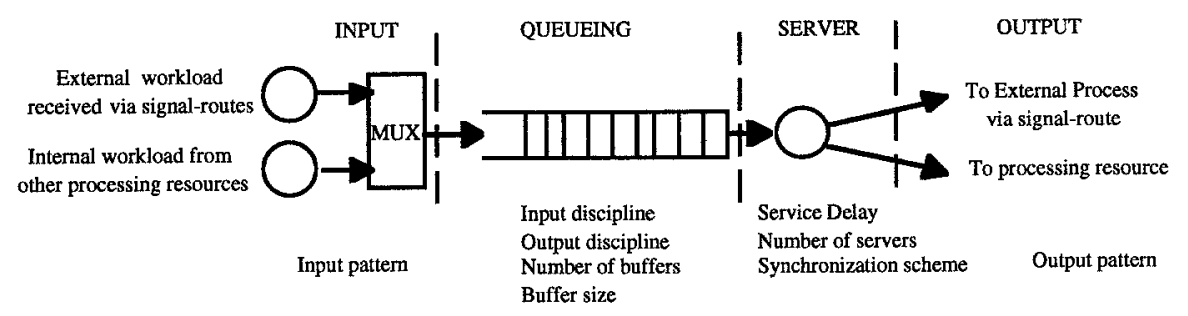

Figure 7: Processing resource

- The input models the arrival of signals to the resource. The signals entering the queue may come from other processes or may have been generated by the process itself. Extended SDL does not require a special description of the input because this information is already provided within the SDL specification.

- The queueing defines the storage policy. The number of queues models the number of distinct data-streams that handle the resource. Several queues are useful to separate signal-streams entering the system. Each queue is described by its queueing discipline, that refers to how the customers are inserted in the queues and selected for service when queues have formed and its buffering capacity (a limited buffering capacity may arise with signal losses). The most common queueing discipline is first in first out (FIFO). Other usual disciplines are last in first out, random service selection or priority based. A queuing discipline is identified by an integer that points to a library.

Each queue identifies the signals that are allowed to enter the queue by a <condition list $>$. If no condition is specified, it means that all signals are allowed. A condition is formed from a signal name and optionally a list of parameter values if required. A priority discipline asserts that signals with the higher priority have to be served first. There are two general situations in priority disciplines: preemptive, where customers with higher priority are allowed to enter service immediately even if customers with lower priority are currently served, and non-preemptive, where high-priority customers 
will be served first but cannot get into service until the customer presently in service is completed. SDL is non-preemptive; it is maintained in Extended SDL.

Potentially infinite buffering capacity is specified by the keyword infinite. Besides, the capacity can be expressed in bits <flag expression $>$ TRUE otherwise in signals.

<queue definition>::= \{queue <queue identifier>(<discipline expression>, <buffer size definition $>[,<$ condition list $>])<$ end $>\}+$

$<$ buffer size definition>::= $[(<$ flag expression $>)]\{<$ buffer size expression> $\mid$ infinite $\}$

$<$ condition list $>::=(<$ signal name $>[:<$ parameter list $>]$ $\{,<$ signal name $>[:<$ parameter list>] $\}^{*}$ )

The server defines how service is provided. The number of servers refers to the number of simultaneous service requests that a processing resource is able to perform. The service time specifies the time required to serve a request. A potentially infinite number of servers is specified by the keyword infinite. Nevertheless an infinite number of servers only is an abstraction that helps to model particular processing configurations. Service is provided according to the automaton specification. The activities to proceed on each signal depend on the state of the process and on the attributes of the signal. Moreover, Extended SDL delay clauses model the processing time of specified actions.

$<$ server definition $>::=$ service $(\{$ number of servers expression $>\mid$ infinite $\})<$ end $>$

The output models the departure of signals. The signals leaving the process resource may depart to another process. The description of the output is already provided within the SDL specification.

\subsubsection{Modeling Operating Load}

Applications (e.g., phone calls, electronic mail, file transfers, or multimedia services) generate demands for service to the system. The model must obtain characteristics for the demands on the target system and then translate those demands into a traffic model. This may be a difficult task because of the dynamic aspect of the service demands traffic. The workload characterization is a crucial part of the performance evaluation model because it is possible to reach misleading conclusions if the workload is not properly selected.

In SDL, the requests at the system's interface are qualitatively described, that is, each communication link identifies which signals are authorized to borrow the link, but there is no quantification. This information is insufficient to establish an adequate model of the workload. In the Extended SDL specification, we enrich the notion of system, by extending it to the original system and its environment. Extended SDL represents the environment by means of a block. Originally, the system corresponds with the environment by means of channels. In Extended SDL these channels connect the "system" to the "environment" block. The structure of the system remains unchanged, while the "environment" block is decomposed into a set of processes (Figure 8). There is exactly one process by channel connection. Each process describes quantitatively the statistical behavior of the interactions between the environment and the original system through a given channel. The information about the nature of the interactions was already contained in the original SDL specification. Thus, Extended SDL only enhances the description by specifying a statistical interarrival and length distribution for each valid signal. The arrival distribution mimics the temporal behavior of the customer producing system requests. The length distribution describes the temporal variation of the signal length. In performance evaluation, the length distribution is used to determine the processing delay of signals because the service 
rate of a processing resource often is expressed in bit/s. Other concerns are not specified in the extension. This extension introduces a set of new processes that model the behavior of the environment, but it does not modify the behavior of the system itself. Therefore we remain consistent with the SDL specification.

$<$ env-block definition $>:=$ block $\{<$ env-block name $>\}<$ end $>$ \{channel to route connection $>\mid<$ entity in block $>$ ]* endblock $[<$ env-block name $>]<e n d>$

$<$ env-process definition $>::=$ envprocess <env-process name $>:<$ channel identifier $>$ $<$ end $>[<$ formal parameters $><$ end $>$ ] $[<$ valid input signal set $>$ ] [<entity in process $>$ \}* $[<$ process body $>][<$ traffic definition $>]$ endprocess $[<$ env-process name $>]<e n d>$

$<$ traffic definition $>::=$ traffic $<$ env-process name $><e n d>\{<$ workload definition $>]\} *$ endtraffic $[<$ env-process name $>]<$ end $>$

$<$ workload definition $>:$ := workload < signal name $><e n d><$ arrival attribute definition $>$ $<$ length attribute definition $>$ [<signal attributes definition $>$ ]

\section{ENVIRONMENT}

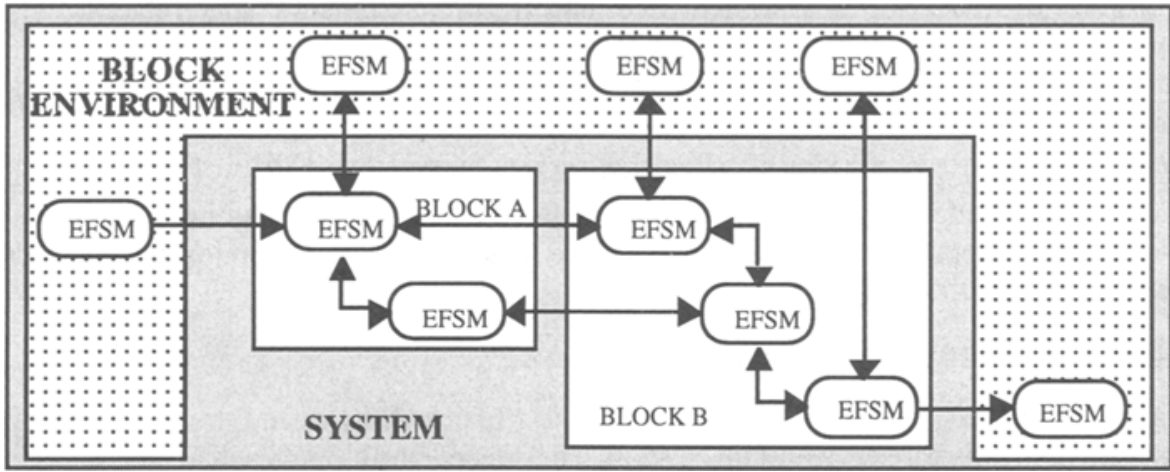

Figure 8: The Extended SDL specification.

\subsubsection{Describing Quality of Service}

A customer sees the system as a black-box to which it applies service requests and from which it gets an output. To achieve satisfactorily service, the customer requires some quality of service (QoS). In [4], the quality of service is defined as a set of userperceivable attributes that make a service what it is. It is expressed in userunderstandable language and manifests itself as a number of parameters, all of which have either subjective or objective values. Objective values are defined and measured in terms of parameters appropriate to the particular service concerned, and which are customer-verifiable. Subjective values are defined and estimated by the provider in terms of the opinion of the customers of the service, collected by means of user surveys. In performance evaluation, the quality of service monitors how the parameters evolve, and checks if the system fits the required values. A quality of service is satisfied when all its performance metrics are satisfied.

In SDL, the specification of the quality of service is missing. Thus, evaluating the performance implies to extend the specification with some static data, which in the performance evaluation environment can be expanded and reused. Since static data declarations do not evolve over time, the addition of the quality of service within Extended SDL does not modify the functional behavior of the specification. $<$ quality of service definition $>::=$ qos $<$ env-service name $><$ end $><$ metrics $>\{,<$ metrics $>$ \} endqos $[<$ env-service name $>]<e n d>$ 
$<$ metrics $>::=<$ delay criteria $>\mid<$ ratio criteria $>\mid<$ utilization criteria $>\mid<j i t t e r s$ criteria $>$ $<$ delay criteria $>::=<$ delay keyword $>$ value $(\{<$ min value $>\mid<\max$ value $>\}[,<$ optimum value $>])<$ end $>$ init $(\{(<$ signal name $>,<$ state name $>,<$ process name $>)\}+)<$ end $>$ close $(\{(<$ signal name $>,<$ state name $>,<$ process name $>)\}+)<$ end $>$

$<$ ratio criteria $>::=<$ ratio keyword $>$ value $(\{<\min$ value $>\mid<\max$ value $>\}[,<$ optimum value $>])<$ end $>$ divisor $\quad(\{(<$ signal name $>,<$ state name $>,<$ process name $>)\}+)$ $<$ end $>$ dividend $(\{(<\underline{\text { signal name }>,<\text { state }}$ name $>,<$ process name $>)\}+)<$ end $>$ $<$ utilization criteria $>::=u$ tilization value $(\{<\underline{\min }$ value $><\max$ value $>\}[,<$ optimum value $>])<$ end $>$ queue $(\{(<$ queue name $>,<$ process name $>)\}+)<$ end $>$

$<$ jitters criteria $>::=$ jitters value $(\{<\underline{\min }$ value $>\mid<\max$ value $>\}[,<\underline{\text { optimum }}$ value $>])$ $<$ end $>$ message $(\{(<$ signal name $>,<$ state name $>,<$ process name $>)\}+)<$ end $>$

$<$ ratio keywords $>::=$ through put|reliabilitylavailabilityllosslerror|resilience $<$ delay keywords $>::=$ activation|releaseltransfer|responselturnaround|reaction

\subsection{Process Mapping - Performance Design Specification}

The principle of the process mapping is to transform each Extended SDL process (EFSM) into a combination of an EFSM and of a queueing network (QN) (Figure 5), where the EFSM models the detailed mechanisms of the process, while the queueing network describes the congestion of multiple requests to restricted resources.

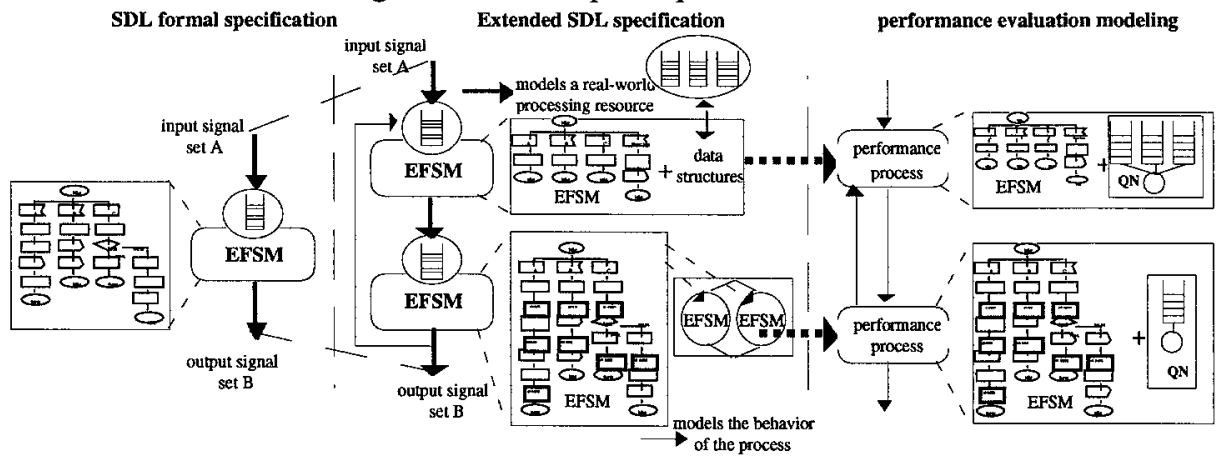

Figure 9: The process mapping technique.

Besides, the process mapping describes the nature and the way in which performance experiments will be conducted. There must be clear objectives of what is expected from the performance evaluation; we should be aware of the goals and limitations of the performance model. We will reuse the quality of service specification which defines what are the relevant metrics to evaluate.

The system may perform the service correctly, incorrectly, or refuse to perform the service. If the system performs the service correctly, its performance is measured by the time taken to perform the service (response time), the rate at which the service is performed (throughput), and the resource rate consumed during service (utilization). This last criterion gives an indication of the percentage of time a resource is busy for a given load. If the system performs the service incorrectly, an error is said to have occurred. It can be helpful to classify errors and to determine the probability of each class of errors. If the system is not able to perform the service, it is said to be unavailable. Two types of performance metrics need to be considered: punctual and global. Punctual metrics are evaluated over a given resource or set of resource, while 
global metrics are evaluated from end to end. There are cases where a decision that optimizes a punctual metric is different from one that optimizes a global metric.

Two types of performance metrics need to be considered: individual and global. Individual metrics reflect the utility of each user, while the global metrics reflect the system wide utility. The resource utilization, reliability, and availability are global metrics, while response time and throughput may be measured for each individual as well as for globally for the system. There are cases where the decision that optimizes individual metrics is different from the one that optimizes the system metric.

\subsection{OPNET - Performance Abstract Implementation}

There are many performance evaluation environments that suit for implementing performance modeling. By our study case we chose a commercial tool: OPtimized Network Engineering Tool (OPNET). OPNET is a simulation environment capable of developing and simulating communication networks with detailed protocol modeling and performance analysis. System behavior and performance are analyzed by performing discrete event simulations. Opnet expresses process models in a language called Proto-C, which is specifically designed to support development of protocols and algorithms. Proto- $\mathrm{C}$ bases on a combination of state transition diagrams, on a library of high level commands called kernel procedures, and on the general facilities of the $C$ programming language. The state diagram description of a process defines a set of primary modes (states) that the process can enter, and the conditions that would cause the process to move from mode to mode. There are other important aspects of Proto$C$; these include actions associated with each mode, variables and attribute declarations, and common expressions and functions. Proto- $\mathrm{C}$ defines two types of modes, called forced and unforced that differ in their execution timing. Unforced modes allow to pause between the enter executives and the exit executives. Forced modes do not allow to wait; therefore they cannot be used to represent modes of the system that persist for any duration. In other words, the exit executives of a forced mode are executed immediately upon completion of the enter executives. The last step consists in verifying whether the customer's quality of service is satisfied or not. When not, we should identify the bottlenecks of the system and optimize them.

\section{A Case Study - Transmission Control Protocol}

Transmission control protocol (TCP) is a transport protocol used among others on Ethernet networks. It provides a connection-oriented, reliable service to the application using it. Two stations using TCP must establish a TCP connection before starting to exchange data. During the exchange of data, TCP packetizes the customer data into segments, sets a time-out any time it sends data, acknowledges data received by the other end, reorders out-of-order data, discards duplicate data, provides end-to-end flow control, and calculates and verifies a mandatory end-to-end checksum. TCP is used by many popular applications, such as Telnet, FTP, or SMTP.

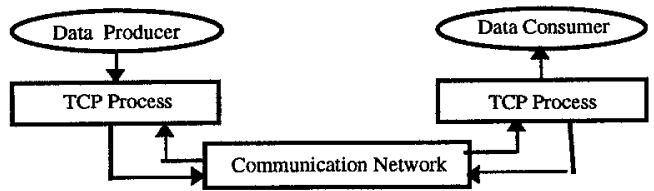

Figure 10: TCP illustration example. 
In this example we focus on the exchange of data; we consider neither the establishment nor the release of TCP connections. Figure 10 shows the outline of the system. "Data Producer" is a customer which generates data that should be reliably transmitted to another customer: "Data Consumer". Between them, there is an unreliable network that generates errors. The TCP process prevents errors by implementing detection and resend mechanisms.

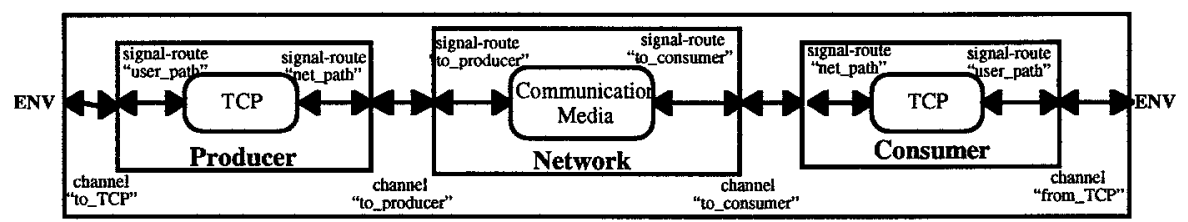

Figure 11: SDL specification of the TCP example.

Figure 11 shows the SDL specification. Customers are not considered. Data enter the system by the channel "to_TCP" and leave by "from_TCP".

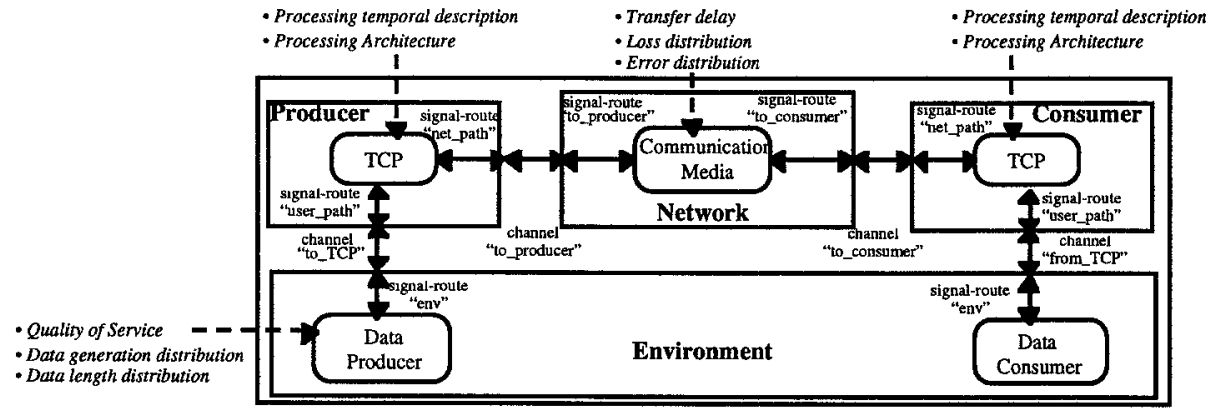

Figure 12: Extended SDL specification of the TCP example.

Figure 12 shows the Extended SDL outline. We enhance the SDL specification with the description of the consumers in terms of quality of service, and of workload (message interarrival and length distributions). We also enhance the description of the TCP processes by describing their processing architecture and their processing constraints. Finally, the network is described in terms of errors.

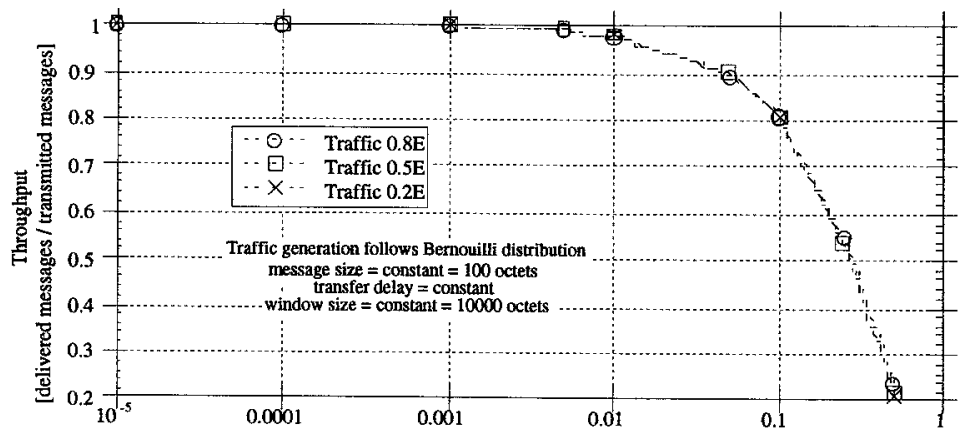

Figure 13: Throughput variation

The QoS states the performance expected from the connection between the "Data Producer" and the "Data Consumer". It embodies the limit values of the delay and of the throughput. These values allow determining if further optimization is necessary. 
During performance experiments, the description of the workloads is used to mimic the generation of messages. Similarly, the processing architecture and the associated processing constraints are used to simulate the non-functional behavior of processes. Finally, the simulation of the system provides information on interesting performance metrics (e.g., resource utilization, delay, losses and throughput), both for end-to-end system and for subsets of the system.

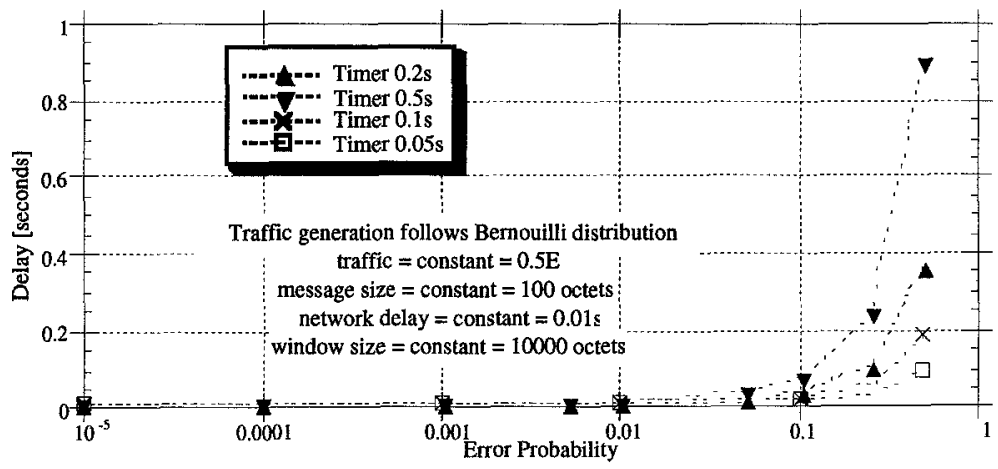

Figure 14: Delay variation as function of the retransmission timer

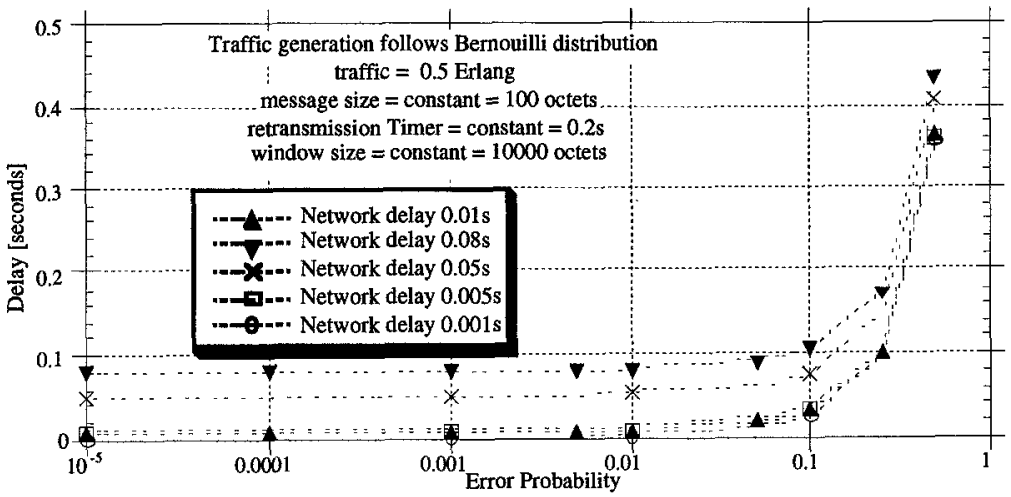

Figure 15: Delay variation as function of the network delay

We have computed simulations of the modeling of our TCP example. This modeling was obtained using our methodology, and was implemented on the tool Opnet. The simulation results (Figure 13,14 and 15) are used to verify under which conditions the required quality of service is fulfilled.

\section{Conclusion}

We believe that modeling performance from formal descriptions is a powerful approach because it saves time by reusing the formal abstraction and avoids errors. Furthermore, it combines the power of formal techniques (validation and conformance testing) with the power of performance evaluation.

Our contribution proposes a methodology reusing the formal specification, enhancing it with performance information and mapping it to a performance modeling. Analyzing the simulation results of the performance modeling allows optimizing the system. We are convinced that our methodology is simple, powerful and generic. It is scalable, that is, it is applicable to simple as well as to complex systems. Besides, 
abstracting real systems first in SDL specifications, results in good validation possibilities that save time and avoid errors in developing a performance modeling. We have no doubt that our methodology can be automated.

\section{Acknowledgments}

The authors are very grateful to Dr. P.-A. Etique from Swiss PTT, and Dr. S. Znaty from EPFL, for their useful expertise and advise. This research was partially funded by the Swiss National Science Foundation.

\section{References}

[1]Bause, Buchholz, Protocol Analysis using a timed version of SDL, In FORTE 1991.

[2]Bolognesi T., Lucidi F.; LOTOS-like Process Algebra with Urgent or Timed Interactions; In IFIP Formal Description Techniques 1991.

[3]Bochmann G.v., Vaucher J., Adding performance aspects to specification languages, In IFIP Protocol Specification, Test and Verification, 1988.

[4]Cochrane; Quality of Service Mappings, In Management of Networks, Ellis 1992.

[5]Dembinski; Queueing Network Model for Estelle; Formal Description Techniques 1993.

[6]Diaz M., Modeling and Analysis of Communication and Cooperation Protocol using Petri Nets based Model, In Protocol Specification, Test and Verification, 1982.

[7]Gouda; Protocol Verification Made Simple;Computer Networks \& ISDN Systems 1993.

[8]Gustafson J. Rudin H.; Including a Queue in a Formal Description driven Protocol Performance Analysis; In Protocol Specification, Test and Verification 1990.

[9]Heck,Müller-Clostermann,Towards the Integration of Formal Description Techniques with Performance Evaluation, Beschreibungstechniken Verteilte Systeme 1992.

[10]Katoen J.-P., Langerak R., Latella D.; Modelling Systems by Probabilistic Process Algebra: An Event Structures Approach; In Formal Description Techniques 1993.

[11]Kritzinger, Wheeler;Semi-Markovian Analysis of Protocol Performance; PSTV 1992.

[12]Leduc G. Léonard L.; A timed LOTOS supporting a dense time domain and including new timed operators; In IFIP Formal Description Techniques 1993.

[13]Leue S.; QoS Specification based on SDLMSC and Temporal Logic; Workshop on Multimedia Applications and Quality of Service Verification, 1994.

[14]Quemada, Azcorra, Frutos, A timed calculus for LOTOS, In FORTE 1989.

[15]Rico,Bochmann; Performance description and analysis for distributed systems using a variant of LOTOS; Protocol Specification, Test and Verification 1991.

[16]Sidhu D.; Protocol Testing: the first ten years, the next ten years; In PSTV 1990.

[17]Sredniawa, Kakol, Gumulinski; SDL in Performance Evaluation; In SDL Forum 1987.

[18]Soerensen,Nordahl,Hansen;From CSP to Markov Models;Trans. Software Eng. 1993.

[19]UIT-T, Z.100; CCITT Specification and Description Language; UIT, 1993.

[20]West C., Protocol Validation-Principles and Applications, Computer Networks \& ISDN Systems 1992.

[21]Wohlin C; Performance Analysis of SDL systems from SDL Descriptions, In SDL Forum III, 1991.

[22]Zhang, Chanson; An Approach to Evaluating the Performance of Communication Protocols based on Formal Specifications; Conference on Network Protocols 1993. 